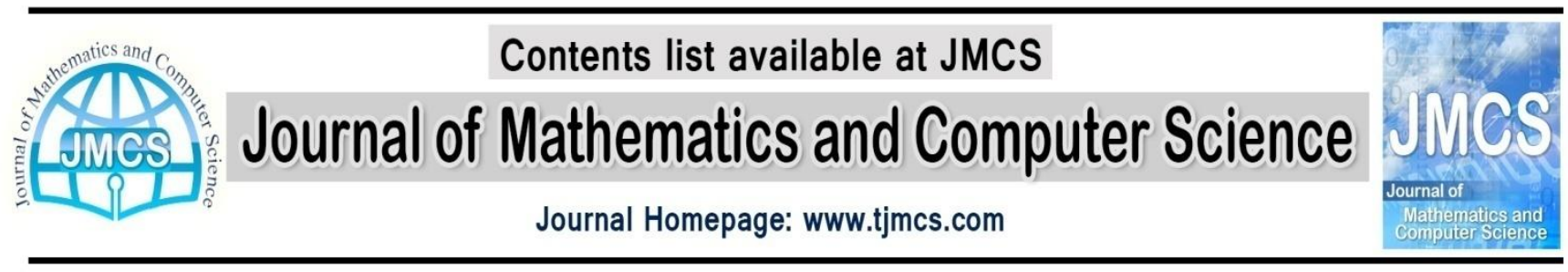

\title{
Fixed Point Theory for Generalized Quasi-contraction Maps in Modular Metric Spaces
}

\author{
Hossein Rahimpoor ${ }^{1, *}$, Ali Ebadian ${ }^{2}$, Madjid Eshaghi Gordji ${ }^{3, *}$, Ali Zohri ${ }^{4}$ \\ ${ }^{1}$ Department of Mathematics, Payame Noor University, P.O. BOX 19395-3697,Tehran, Iran \\ ${ }^{2}$ Department of Mathematics, Faculty of Science, Urmia University, Urmia, Iran \\ ${ }^{3}$ Department of Mathematics, P.O.Box 35195-363, Semnan, Iran \\ ${ }^{4}$ Department of Mathematics, Payame Noor University, P.O. BOX 19395-3697, Tehran, Iran \\ E-mail: rahimpoor2000@yahoo.com ; E-mail: ebadian.ali@gmail.com \\ E-mail: madjid.eshaghi@gmail.com ; E-mail: alizohri@gmail.com
}

\begin{abstract}
*The corresponding authors: rahimpoor2000@yahoo.com (H. Rahimpoor) and madjid.eshaghi@ gmail.com (M. Eshaghi Gordji)
Article history:

Received January 2014

Accepted March 2014

Available online March 2014
\end{abstract}

\section{Abstract}

In this paper, we improve the results of the existence of fixed point theory for generalized quasi contraction maps in modular metric spaces which extends the results of Y.Cho.et al,[Quasi-contraction mapping in modular metric spaces, Journal of Applied Mathematics Volume 2012 (2012), Article ID 907951, 5 pages] .

Keywords: modular metric spaces, quasi-contraction mapping, fixed point .

\section{Introduction}

The theory of modular spaces was initiated by Nakano in 1950 [12], and generalized and redefined by Musielak and Orlicz [[10],[11]] in 1959. In 2008, Chistyakov [7] introduced the notation of modular metric spaces generated by Fmodular and develop the theory of modular spaces, by the same idea he was 
defined the modular metric spaces on an arbitrary set which is a new generalization of metric spaces[[4],[5]].

Many mathematicians studied fixed point results for modular metric spaces, see ([6],[9],[2],[1]).In 1974

Çiriç introduced quasi-contraction maps and proved a fixed point theorem for this kind of maps in complete metric spaces [8].

Recently Y.J.Cho [3] proved a fixed point theory for quasi-contraction maps in modular metric spaces. In this paper, we investigated a fixed point result for generalized quasicontraction maps in this spaces, that extends the results of Y. J. Cho [3].

Let $X$ be a nonempty set, A function $\omega:(0,1) \times X \times X \rightarrow[0, \infty]$ that will be written as $\omega_{\lambda}(x, \mathrm{y})=\omega(\lambda, \mathrm{x}, y)$ is said to be a (metric) pesudomodular on $X$, if it satisfies the following conditions:

(i) given $x, y \in X, \omega_{\lambda}(x, y)=0$ for all $\lambda>0$ if and only if $x=y$;

(ii) $\omega_{\lambda}(x, y)=\omega_{\lambda}(y, x)$, for all $\lambda>0$ and $x, y \in X$;

(iii) $\omega_{\lambda+\mu}(x, y) \leq \omega_{\lambda}(x, z)+\omega_{\mu}(z, y)$ for all $\lambda, \mu>0$ and $x, y, z \in X$.

If instead of $(i)$, we have only the condition

$\left(i_{1}\right) \omega_{\lambda}(x, x)=0$, then $\omega$ is said to be a (metric) pseudomodular on $X$ and if $\omega$ satisfies $\left(i_{1}\right)$

and

$\left(i_{2}\right)$ given $x, y \in X$, if there exists $\lambda>0$, possibly depending on $x$ and $y$ such that $\omega_{\lambda}(x, y)=0$

then $x=y$,

then $\omega$ is called a strict modular on $X$.

Definition 1.1. [4] Given a modular $\omega$ on a set $X$, the sets

$$
X_{\omega} \equiv X_{\omega}\left(x_{\circ}\right)=\left\{x \in X: \omega_{\lambda}\left(x, x_{\circ}\right) \rightarrow 0 \text { as } \lambda \rightarrow \infty\right\}
$$

and

$$
X_{\omega}^{*} \equiv X_{\omega}^{*}\left(x_{\circ}\right)=\left\{x \in X: \omega_{\lambda}\left(x, x_{\circ}\right)<\infty \text { for some } \lambda>0\right\}
$$

is said to be modular space (around $x_{\circ}$ )Also the modular space $X_{\omega}$ and $X_{\omega}^{*}$ can be equipped with metrics $d_{\omega}$ and $d_{\omega}^{*}$, generated by $\omega$ and given by

$$
d_{\omega}(x, y)=\inf \left\{\lambda>0: \omega_{\lambda}(x, y) \leq \lambda\right\}, \quad x, y \in X_{\omega}
$$

and

$$
d_{\omega}^{*}(x, y)=\inf \left\{\lambda>0: \omega_{\lambda}(x, y) \leq 1\right\}, \quad x, y \in X_{\omega}^{*}
$$

Remark 1.2. Given a modular $\omega$ on a set $X$, the function $0<\lambda \mapsto \omega_{\lambda}(x, y) \in[0, \infty]$ for given $x, y \in X$, is non-increasing on $(0, \infty)$. Indeed,

if $0<\lambda<\mu$, then we have

$$
\omega_{\mu}(x, y) \leq \omega_{\mu-\lambda}(x, x)+\omega_{\lambda}(x, y)=\omega_{\lambda}(x, y)
$$

for all $x, y \in X$. 
Definition 1.3. [6] A sequence $\left\{x_{n}\right\} \equiv\left\{x_{n}\right\}_{n=1}^{\infty}$ from $X_{\omega}$ is said to be $\omega$-convergent to an element $x \in X$ if there exists a number $\lambda_{0}>0$, possibly depending on $\left\{x_{n}\right\}$ and $x$, such that

$$
\lim _{n \rightarrow \infty} \omega_{\lambda_{0}}\left(x_{n}, x\right)=0 \text { or } x_{n} \stackrel{\omega}{\longrightarrow} x(\text { as } n \rightarrow \infty) \text {. }
$$

Definition 1.4. [6]A sequence in $X_{\omega}$ is said to be $\omega$-Cauchy if there exists a number $\lambda_{0}=\lambda_{0}\left\{x_{n}\right\}>0$ and $n_{\circ}(\varepsilon) \in \mathrm{N}$ such that for all $n, m \geq n_{\circ}(\varepsilon)$ we have

$$
\omega_{\lambda_{0}}\left(x_{n}, x_{m}\right) \leq \varepsilon
$$

Definition 1.5. [6] The modular space $X_{\omega}$ is said to be $\omega$-complete if any $\omega$-Cauchy sequence from $X_{\omega}$ is $\omega$-convergent and its limit belong to $X_{\omega}$.

Definition 1.6. Given a modular $\omega$ on $X$ a subset $C \subseteq X_{\omega}$ is said to be $\omega$-closed if for each sequence $\left\{x_{n}\right\} \in C$ with $x_{n} \stackrel{\omega}{\longrightarrow} x$, we have $x \in C$.

Let $\Pi$ be the class of all maps $\pi:[0, \infty) \cup\{\infty\} \rightarrow[0, \infty) \cup\{\infty\}$ with the following conditions :

(1) $\pi$ is non-decreasing;

(2) for each $t \in[0, \infty), \pi(t)<\infty$ and

$$
\lim _{n \rightarrow \infty}\left(\sup \left\{\pi^{n}(t), \pi^{n+1}(t), \pi^{n+2}(t), \ldots\right\}\right)=0
$$

(3) $\left.\pi\right|_{[0, \infty)} \in \Pi$

Definition 1.7. Given a modular $\omega$ on $X$, we say $\omega$ has Fatou property if and only if

$$
\omega_{\lambda}(x, y) \leq \liminf _{n \rightarrow \infty} \omega_{\lambda}\left(x_{n}, y\right) \text { for all } \lambda>0 \text { and } y \in X_{\omega} .
$$

where $x_{n} \stackrel{\omega}{\longrightarrow} x$ (as $\left.n \rightarrow \infty\right)$.

\section{Main result}

In this section we define the concept of generalized quasi-contraction and then we prove the main result.

Definition 2.1. Let $X_{\omega}$ be a modular metric spaces. Let $C$ be a nonempty subset of $X_{\omega}$. The self map $T: C \rightarrow C$ is said to be generalized quasi-contraction if there exists a $\pi \in \Pi$ such that

$$
\omega_{\lambda}(T x, T y) \leq P_{\pi}(x, y),
$$

where

$$
P_{\pi}(x, y)=\sup \left\{\pi\left(\omega_{\lambda}(x, y)\right), \pi\left(\omega_{\lambda}(x, T x)\right), \pi\left(\omega_{\lambda}(y, T y)\right), \pi\left(\omega_{\lambda}(x, T y)\right), \pi\left(\omega_{\lambda}(T x, y)\right)\right\}
$$

for any $x, y \in C$ and $\lambda \in(0, \infty)$.

Definition 2.2. Let $T, C$ be as in the above definition. For any $x \in C$, define the orbit

$$
\mathrm{O}(x)=\left\{x, T x, T^{2} x, \ldots\right\}
$$


and it's $\omega$-diameter by

$$
\delta_{\omega}(x)=\operatorname{diam}(\mathrm{O}(x))=\sup \left\{\omega_{\lambda}\left(T^{n} x, T^{m} x\right) ; n, m=0,1,2, \ldots\right\}
$$

We need the following lemma in the main theorem of the present paper.

Lemma 2.3. Let $X_{\omega}$ be a modular metric space and let $C$ be a nonempty subset of $X_{\omega}$.Let $T: C \rightarrow C$ be generalized quasi-contraction and $x \in C$ be such that $\delta_{\omega}(x)<\infty$. Then for any $n \geq 1$ we have

$$
\delta_{\omega}\left(T^{n} x\right) \leq \pi^{n}\left(\delta_{\omega}(x)\right)
$$

moreover one has

$$
\omega_{\lambda}\left(T^{n} x, T^{n+m} x\right) \leq \pi^{n}\left(\delta_{\omega}(x)\right)
$$

for all $n \geq 1, m \geq 0$.

Proof. Let $m \geq n \geq 1$, we have

$$
\omega_{\lambda}\left(T^{n} x, T^{m} y\right)=\omega_{\lambda}\left(T\left(T^{n-1} x\right), T\left(T^{m-1} y\right)\right) \leq P_{\pi}\left(T^{n-1} x, T^{m-1} y\right)
$$

this implies for $m, n \geq 1$

$\omega_{\lambda}\left(T^{n} x, T^{m} y\right)=\sup \left\{\pi\left(\omega_{\lambda}(x, y)\right), \pi\left(\omega_{\lambda}(x, T x)\right), \pi\left(\omega_{\lambda}(y, T y)\right), \pi\left(\omega_{\lambda}(x, T y)\right), \pi\left(\omega_{\lambda}(T x, y)\right)\right\}$ and so

$$
\delta_{\omega}\left(T^{n} x\right) \leq \pi\left(\delta_{\omega}\left(T^{n-1} x\right)\right) \text { for any } n \geq 1 .
$$

Therefore for any $n \geq 1$ we have

$$
\delta_{\omega}\left(T^{n} x\right) \leq \pi^{n}\left(\delta_{\omega}(x)\right) .
$$

Moreover for any $n \geq 1$ and $m \in\{0,1,2, \ldots\}$ we have

$$
\omega_{\lambda}\left(T^{n} x, T^{n+m} x\right) \leq \delta_{\omega}\left(T^{n}(x)\right) \leq \pi^{n}\left(\delta_{\omega}(x)\right) .
$$

Theorem 2.4. Let $X_{\omega}$ be a modular metric space such that $\omega$ satisfies the Fatou property and $C$ be a nonempty $\omega$-complete subspace of $X_{\omega}$. Let $T: C \rightarrow C$ be generalized quasicontraction and $x \in C$ be such that $\delta_{\omega}(x)<\infty$. Then the $T^{n} x$ is $\omega$-convergent to $u$ that is fixed point of $T$. Moreover if $u^{*}$ is any fixed point of $T$ in $C$ such that $\omega_{\lambda}\left(u, u^{*}\right)<\infty$, then $u=u^{*}$.

Proof. We know that

$$
0 \leq \pi^{n}(t) \leq \sup \left\{\pi^{n}(t), \pi^{n+1}(t), \pi^{n+2}(t), \ldots\right\}
$$

and

$$
\lim _{n \rightarrow \infty} \sup \left\{\pi^{n}(t), \pi^{n+1}(t), \pi^{n+2}(t), \ldots\right\}=0
$$

SO 


$$
\lim _{n \rightarrow \infty} \pi^{n}(t)=0 \text { for each } t \in[0, \infty)
$$

Hence by Lemma $2.3\left\{T^{n} x\right\}$ is $\omega$-Cauchy sequence. Since $C$ is $\omega$-complete so there exists $u \in C$ such that $T^{n} x \stackrel{\omega}{\longrightarrow} u$ (as $\left.n \rightarrow \infty\right)$, so by Fatou property we get

$$
\omega_{\lambda}\left(T^{n} x, u\right) \leq \liminf _{n \rightarrow \infty} \omega_{\lambda}\left(T^{n} x, T^{n+m} x\right) \leq \pi^{n}\left(\delta_{\omega}(x)\right) \text { for any } n \geq 0 .
$$

By assumption $T$ is generalized quasi-contraction, so

$$
\omega_{\lambda}(T x, T u) \leq P_{\pi}(x, u) .
$$

Since $\omega$ is non-decreasing from (2.1) we get

$$
\omega_{\lambda}(T x, T u) \leq \sup \left\{\pi\left(\delta_{\omega}(x)\right), \pi^{2}\left(\delta_{\omega}(x)\right), \pi\left(\omega_{\lambda}(u, T u)\right), \pi\left(\omega_{\lambda}(x, T u)\right)\right\}
$$

and

$$
\omega_{\lambda}(T x, T u) \leq \sup \left\{\pi\left(\omega_{\lambda}(x, u)\right), \pi\left(\omega_{\lambda}(x, T x)\right), \pi\left(\omega_{\lambda}(u, T u)\right), \pi\left(\omega_{\lambda}(x, T u)\right), \pi\left(\omega_{\lambda}(T x, u)\right)\right\}
$$

so

$$
\omega_{\lambda}(T x, T u) \leq \sup \left\{\pi\left(\delta_{\omega}(x)\right), \pi^{2}\left(\delta_{\omega}(x)\right), \pi\left(\omega_{\lambda}(u, T u)\right), \pi\left(\omega_{\lambda}(x, T u)\right)\right\}
$$

by induction for $n \geq 1$ we get

$$
\omega_{\lambda}\left(T^{n} x, T u\right) \leq \sup \left\{\pi^{n}\left(\delta_{\omega}(x)\right), \pi^{n+1}\left(\delta_{\omega}(x)\right), \pi\left(\omega_{\lambda}(u, T u)\right), \pi^{2}\left(\omega_{\lambda}(u, T u)\right), \ldots, \pi^{n}\left(\omega_{\lambda}(u, T u)\right), \pi^{n}\left(\omega_{\lambda}(x, T u)\right)\right\} .
$$

Indeed;

$$
\begin{aligned}
& \omega_{\lambda}\left(T^{n+1} x, T u\right)=\omega_{\lambda}\left(T^{n}(T x), T u\right) \\
& \leq \sup \left\{\pi^{n}\left(\delta_{\omega}(T x)\right), \pi^{n+1}\left(\delta_{\omega}(T x)\right), \pi\left(\omega_{\lambda}(u, T u)\right), \ldots, \pi^{n}\left(\omega_{\lambda}(u, T u)\right), \pi^{n}\left(\omega_{\lambda}(T x, T u)\right)\right\} \\
& \leq \sup \left\{\pi^{n+1}\left(\delta_{\omega}(T x)\right), \pi^{n+2}\left(\delta_{\omega}(T x)\right), \pi\left(\omega_{\lambda}(u, T u)\right), \ldots, \pi^{n+1}\left(\omega_{\lambda}(u, T u)\right), \pi^{n+1}\left(\omega_{\lambda}(T x, T u)\right)\right\}
\end{aligned}
$$

observe that for $n \geq 1$ we have

$\omega_{\lambda}\left(T^{n} x, T u\right) \leq \pi^{n}\left(\delta_{\omega}(x)\right)+\pi^{n+1}\left(\delta_{\omega}(x)\right)+\pi^{n}\left(\omega_{\lambda}(x, T u)\right)+\sup \left\{\pi\left(\omega_{\lambda}(u, T u)\right), \pi^{2}\left(\omega_{\lambda}(u, T u)\right), \ldots, \pi^{n}\left(\omega_{\lambda}(u, T u)\right)\right\}$ that yields

$$
\begin{aligned}
\varlimsup_{n \rightarrow \infty} \omega_{\lambda}\left(T^{n} x, T u\right) & \leq \varlimsup_{\lim } \pi^{n}\left(\delta_{\omega}(x)\right)+\varlimsup_{n \rightarrow \infty} \pi^{n+1}\left(\delta_{\omega}(x)\right)+\varlimsup_{n \rightarrow \infty} \pi^{n}\left(\omega_{\lambda}(x, T u)\right) \\
& +\varlimsup_{n \rightarrow \infty}\left\{\pi\left(\omega_{\lambda}(u, T u)\right), \pi^{2}\left(\omega_{\lambda}(u, T u)\right), \ldots, \pi^{n}\left(\omega_{\lambda}(u, T u)\right)\right\} \\
& =\varlimsup_{n \rightarrow \infty} \sup \left\{\pi\left(\omega_{\lambda}(u, T u)\right), \pi^{2}\left(\omega_{\lambda}(u, T u)\right), \ldots, \pi^{n}\left(\omega_{\lambda}(u, T u)\right)\right\} \\
& \leq \sup \left\{\pi\left(\omega_{\lambda}(u, T u)\right), \pi^{2}\left(\omega_{\lambda}(u, T u)\right), \ldots, \pi^{n}\left(\omega_{\lambda}(u, T u)\right), \ldots\right\} .
\end{aligned}
$$

By Fatou property we have

$$
\begin{aligned}
\omega_{\lambda}(u, T u) & \leq \liminf _{n \rightarrow \infty} \omega_{\lambda}\left(T^{n} x, T u\right) \\
& \leq \varlimsup_{n \rightarrow \infty} \omega_{\lambda}\left(T^{n} x, T u\right) \\
& \leq \sup \left\{\pi\left(\omega_{\lambda}(u, T u)\right), \pi^{2}\left(\omega_{\lambda}(u, T u)\right), \ldots, \pi^{n}\left(\omega_{\lambda}(u, T u)\right), \ldots\right\}
\end{aligned}
$$


Therefore

$$
\pi\left(\omega_{\lambda}(u, T u)\right) \leq \sup \left\{\pi^{2}\left(\omega_{\lambda}(u, T u)\right), \pi^{3}\left(\omega_{\lambda}(u, T u)\right), \ldots, \pi^{n}\left(\omega_{\lambda}(u, T u)\right), \ldots\right\}
$$

hence

$$
\omega_{\lambda}(u, T u) \leq \sup \left\{\pi^{2}\left(\omega_{\lambda}(u, T u)\right), \pi^{3}\left(\omega_{\lambda}(u, T u)\right), \ldots, \pi^{n}\left(\omega_{\lambda}(u, T u)\right), \ldots\right\}
$$

so by induction

$$
\omega_{\lambda}(u, T u) \leq \sup \left\{\pi^{n}\left(\omega_{\lambda}(u, T u)\right), \pi^{n+1}\left(\omega_{\lambda}(u, T u)\right), \ldots\right\}
$$

so

$$
\omega_{\lambda}(u, T u) \leq \limsup _{n \rightarrow \infty}\left\{\pi^{n}\left(\omega_{\lambda}(u, T u)\right), \pi^{n+1}\left(\omega_{\lambda}(u, T u)\right), \ldots\right\}=0 .
$$

Therefore $\omega_{\lambda}(u, T u)=0$, that yields $u=T u$.

Now if $u^{*}$ be another fixed point that $\omega_{\lambda}\left(u, u^{*}\right)<\infty$ then

$$
\omega_{\lambda}\left(u, u^{*}\right)=\omega_{\lambda}\left(T u, T u^{*}\right) \leq P_{\pi}\left(u, u^{*}\right)=\pi\left(\omega_{\lambda}\left(u, u^{*}\right)\right)
$$

so

$$
\omega_{\lambda}\left(u, u^{*}\right) \leq \pi\left(\omega_{\lambda}\left(u, u^{*}\right)\right) \leq \pi^{2}\left(\omega_{\lambda}\left(u, u^{*}\right)\right) \leq \ldots
$$

by induction

$$
\omega_{\lambda}\left(u, u^{*}\right) \leq \pi^{n}\left(\omega_{\lambda}\left(u, u^{*}\right)\right)
$$

so $\omega_{\lambda}\left(u, u^{*}\right)=0$, hence $u=u^{*}$.

\section{References}

[1] P. Chaipunya, Y.J.Cho, P. Kumam, Geraghty-type theorems in Modular Metric Spaces with an application to partial differential equation, Fixed point theory and applications 2012,2012:83 doi : $10.1186 / 1687-1847-2012-83$

[2] P. Chaipunya, C. Mongkolkeha, W. Sintunavarat, P. Kumam, Fixed-Point Theorems for Multivalued Mappings in Modular Metric Spaces, Abstract and Applied Analysis Volume 2012 (2012), Article ID 503504, 14 pagesdoi:10.1155/2012/503504

[3] Y. Cho, R. Saadati, G. Sadeghi, Quasi-contraction mapping in modular metric spaces, Journal of Applied Mathematics Volume 2012 (2012), Article ID 907951, 5 pages doi:10.1155/2012/907951

[4] V.V Chistyakov, Modular metric spaces, I:Basic concepts, Nonlinear Analysis, 72(2010)1-14.

[5] V.V Chistyakov, Modular metric spaces, II:Application to superposition operators, Nonlinear Analysis, 72(2010)15-30.

[6] V.V Chistyakov, A fixed point theorem for contractions in modular metric spaces, Perprint submited to $\operatorname{arXiv}(2011)$. 
[7] V. V. Chystyakov, Modular metric spaces generated by F-modulars, Folia Math. 15 (1) (2008) 3-24. MR2646913 (2011j:54030)

[8] L. B. Çiriḉ, A generalization of Banachs contraction principle, Proceedings of the American Mathematical Society 45(2)(1974)267-27

[9] C. Mongkolkeha, W. Sintunavarat, P. Kumam, Fixed point theorem for contraction mappings in modular metric spaces, Fixed Point Theory and Applications 2011,2011 :93 doi:10.118611687-18122011-93.

[10] J. Musielak, W. Orlicz, On modular spaces, Studia Mathematica 18 (1959) 49-65.

[11] J. Musielak, W. Orlicz, Some remarks on modular spaces,Bull. Acad. Polon. Sci. Sr. Sci. Math. Astron. Phys. 7, 661-668(1959).

[12] H. Nakano, Modular semi-ordered spaces, Tokyo, 1950. 